\title{
Glass Transition of Disentangled and Entangled Polymer Melts: Single-Chain-Nanoparticles Approach
}

Manjesh K. Singh, Minghan Hu, Yu Cang, Hsiao-Ping Hsu, Heloise Therien-Aubin, Kaloian Koynov, George Fytas, Katharina Landfester, and Kurt Kremer*

Cite This: Macromolecules 2020, 53, 7312-7321

Read Online

ABSTRACT: We study the effect of entanglements on the glass transition of high molecular weight polymers, by the comparison of single-chain nanoparticles (SCNPs) and equilibrated melts of highmolecular weight polystyrene of identical molecular weight. SCNPs were prepared by electrospraying technique and characterized using scanning electron microscopy and atomic force microscopy techniques. Differential scanning calorimetry, Brillouin light spectroscopy, and rheological experiments around the glass transition were compared. In parallel, entangled and disentangled

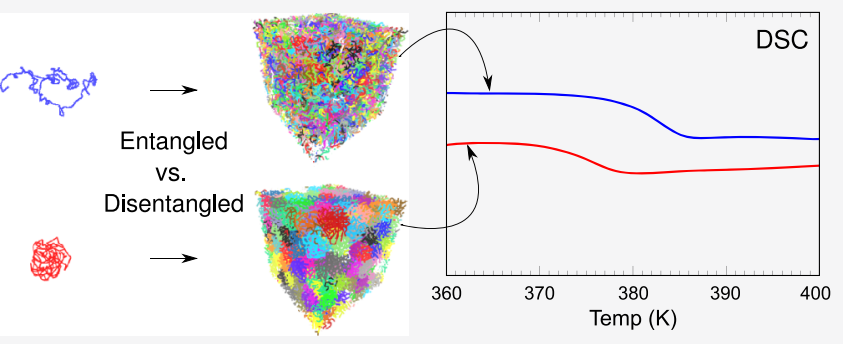
polymer melts were also compared under cooling from molecular dynamics simulations based on a bead-spring polymer model. While experiments suggest a small decrease in the glass transition temperature of films of nanoparticles in comparison to entangled melts, simulations do not observe any significant difference, despite rather different chain conformations.

\section{INTRODUCTION}

There has been a great interest to understand the relation between glass transition temperature $\left(T_{\mathrm{g}}\right)$ and molecular weight $\left(M_{n}\right)$ of polymers. ${ }^{1-8}$ For polystyrene (PS), $T_{g}$ significantly increases with $M_{\mathrm{n}}$ slowly leveling off around the entanglement molecular weight $\left(M_{\mathrm{e}}\right)$. For short polymer chains of only a few monomers, conformations are still approaching the asymptotic Gaussian structure with increasing chain length, while for molecular weight around $M_{\mathrm{e}}$ conformations typically are reasonably well represented by a Gaussian coil structure. Thus, beyond $M_{\mathrm{e}}, T_{\mathrm{g}}$ does not change much with increasing $M_{\mathrm{n}}$ and approaches a constant value of about $T_{\mathrm{g}}=374 \mathrm{~K}$ for high molecular weight PS. $^{7}$ It is this regime, where entanglements clearly dominate the viscoelastic response of a melt, we are focusing on in the present work.

In general, the relationship between $T_{\mathrm{g}}$ and $M_{\mathrm{n}}$ can be expressed in terms of modified version ${ }^{7}$ of the well-known Fox and Flory equation: ${ }^{1,2}$

$$
\frac{1}{T_{\mathrm{g}}\left(M_{\mathrm{n}}\right)}=\frac{1}{T_{\mathrm{g}}(\infty)}+\frac{K_{\mathrm{c}}}{M_{\mathrm{n}}}
$$

where $T_{\mathrm{g}}(\infty)$ is the extrapolated $T_{\mathrm{g}}$ of a polymer at infinite $M_{\mathrm{n}}$ and $K_{c}$ is an experimentally determined parameter, depending on the particular polymer species. For PS, $K_{\mathrm{c}}=0.78 \mathrm{Da} / \mathrm{K}$ and the asymptotic value of $T_{\mathrm{g}}(\infty)=374 \mathrm{~K}^{7}$ To rationalize this continued chain length dependency $\left(T_{\mathrm{g}}-M_{\mathrm{n}}\right.$ relationship) beyond $M_{\mathrm{e}}$, two lines of arguments can be found in the literature. The first one proposes that entanglements are central for the remaining shift in and physical properties near
$T_{\mathrm{g}}{ }^{9,10}$ The alternative explanation is that chain-ends are responsible for the observed behavior. The end groups have higher mobility because of their local excess free volume resulting in larger conformational entropy and deficient intermolecular constraints. Lower $M_{\mathrm{n}}$ means higher concentration of chain ends and hence higher mobility leading to lower $T_{\mathrm{g} .}{ }^{11-15}$ Thus, the question we are addressing is to what extent do entanglements play a role for the glass transition. To do this, we have to look for systems identical up to the presence of entanglements. For this, we study experimentally and by simulation equilibrated polymer melts and systems made of disentangled single-chain nanoparticles (SCNPs).

SCNPs can be used to study the effect of entanglements on the properties of long chain polymer melts. Heating SCNPs of high molecular weight above $T_{\mathrm{g}}$ leads to the formation of a metastable disentangled melt of polymers. Their corresponding properties can be compared to that of an entangled melt of bulk polymers. ${ }^{16-18}$ SCNPs can be prepared using a variety of techniques such as precipitation, ${ }^{19-22}$ freeze-drying, ${ }^{16,23,24}$ crystallization, ${ }^{17,18}$ spin-casting, ${ }^{25}$ and electrospraying. ${ }^{26-28}$ All these preparation methods have the use of very dilute polymer solutions in common. In electrospraying, electric

Received: March 10, 2020

Revised: July 7, 2020

Published: August 20, 2020 
fields are applied to facilitate the production of extremely small droplets out of polymer solutions, almost exclusively containing only one polymer chain each. The solvents evaporate, and isolated particles of single polymer molecules are collected. SCNPs can also be used as molecular mass and size standard, biological tracer and to study nano- and microphases where each particle can be considered as a distinct phase. $^{27}$

Previous works ${ }^{9,10}$ on the effect of entanglement near $T_{\mathrm{g}}$ are not consistent. Rong et al. ${ }^{9}$ show a decrease in the $T_{\mathrm{g}}$ of PS $\left(M_{\mathrm{n}}=3.18 \times 10^{5} \mathrm{Da}\right)$ in disentangled freeze-dried dilute solution of PS in benzene. However, Zheng and Simon ${ }^{29}$ used the same approach and concluded that the complete removal of benzene is not possible under those conditions. Hence, the reported ${ }^{9} T_{\mathrm{g}}$ decrease could be ascribed to a solvent-induced plasticization and not to the absence of entanglements. Huang et al. ${ }^{10}$ studied the effect of entanglements on the $T_{\mathrm{g}}$ of lowmolecular-weight polycarbonates with a mass-average molecular weight $M_{\mathrm{w}}=1.7 \times 10^{4} \mathrm{Da}$ and polydispersity $M_{\mathrm{w}} / M_{\mathrm{n}}=$ 1.79. Because polycarbonate has an entanglement molecular weight $M_{\mathrm{e}} \approx 2500 \mathrm{Da},{ }^{30}$ the studied polycarbonate chains have $z=M_{\mathrm{n}} / M_{\mathrm{e}} \leq 5$. Considering this and the rather significant polydispersity make it difficult to clearly identify the cause of the reduction in $T_{\mathrm{g}}$.

In this work, we study PS melts around $T_{\mathrm{g}}$ consisting of disentangled, collapsed high molecular weight single polymer chain nanoparticles (NPs) and compare their properties to the corresponding equilibrated melts. Samples were prepared by electrospraying dilute polymer solutions of PS in dimethylformamide (DMF). The objective of the sample preparation was to minimize entanglements in the melt of long polymer chains. For this, the polymers were dissolved at dilute concentration and then electrosprayed. By working at a concentration well below the overlap concentration $\left(C<C^{*}\right)$, solutions essentially without any entanglements have been prepared. Then, by electrospraying, rapidly drying solution droplets were produced which led to the formation of NPs, containing ideally only single chains, having no or only very few entanglements. The idea was that with the application of high voltage, the initially larger droplets containing polymer and solvent would break-up into smaller drops containing single polymer chains only, and the solvent would evaporate so quickly that only dry polymers in their collapsed initial conformation would be collected. The time to reach the collector $(t \approx 7 \mathrm{~ms})$ was orders of magnitude less than the estimated Rouse-time ${ }^{31,32}\left(\tau_{\mathrm{R}} \approx 1.5 \mathrm{~s}\right)$ of the polymer chains (details in Section S3 of Supporting Information). The low concentration $\left(C<C^{*}\right)$ ensures no interchain entanglements, and the fast evaporation also guaranties no intrachain entanglements (beyond possible very rare intrachain entanglements already existing in the dilute solution). We used different characterization tools like nuclear magnetic resonance (NMR), Fourier-transform infrared spectroscopy and thermal gravimetric analysis (TGA) to rule out the presence of residual DMF in the samples (spectra and graphs in Sections S2.2.2, S2.2.3, and S2.2.4 of the Supporting Information). We applied three different experimental techniques, differential scanning calorimetry (DSC), oscillatory rheology, and Brillouin light scattering (BLS) to analyze melts of disentangled polymer globules in comparison to entangled PS melts. To complement this and to obtain complementary insights into structural aspects, we further employed molecular dynamics (MD) simulations of entangled and disentangled polymer melts. At first glance, our experi- ments give consistent results of a somewhat lower $T_{\mathrm{g}}$ for the disentangled polymer melt in comparison to the entangled polymer melt, while simulations do not support this shift. Different experimental techniques measure $T_{\mathrm{g}}$ by tracking different dynamical properties during the transition. This can lead to somewhat different absolute $T_{\mathrm{g}}$ values. However, our study is aimed at examining deviations in properties around $T_{\mathrm{g}}$ between disentangled and entangled state of polymer melts but is not focusing on the absolute value of $T_{\mathrm{g}}$ itself.

\section{NP PREPARATION}

We have used electrospraying to prepare single-chain PS NPs. Chains of $M_{n}=1.1 \times 10^{6} \mathrm{Da}$ having polydispersity of $M_{\mathrm{w}} / M_{\mathrm{n}}=$ 1.27 were used for our experiments, for more details, we refer to Supporting Information. Different morphologies were obtained by electrospraying polymer solutions of PS in DMF at different concentrations, near, above, and below $C^{*}$. Figure 1 illustrates the electrospraying technique showing high-speed

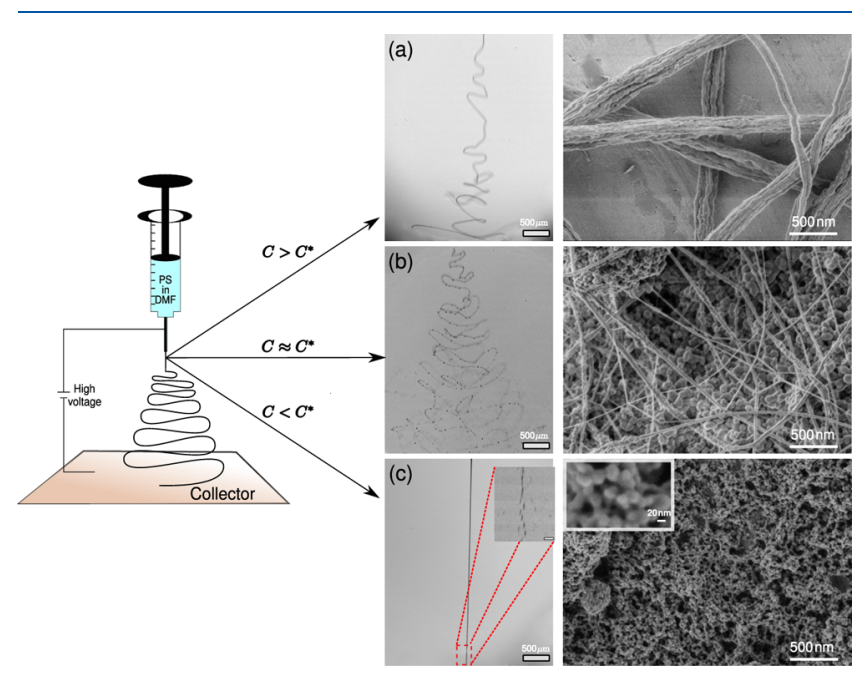

Figure 1. Optimization of the electrospraying process. Samples prepared using electrospraying of PS in DMF solution at concentrations: (a) $C=2.3 \mathrm{wt} \%$, (b) $C=0.5 \mathrm{wt} \%$, and (c) $C=$ 0.1 wt $\%$. The scale bar in the inset corresponds to $50 \mu \mathrm{m}$. Electrospraying was performed under the following process parameters, distance $d=20 \mathrm{~cm}$, infuse-rate $v=1.5 \mathrm{~mL} / \mathrm{h}$, and applied voltage $V=15 \mathrm{kV}$.

camera images of jets of polymer solutions coming out of the needle and scanning electron microscopy (SEM) images of different morphologies obtained at different concentrations. Details of materials and characterizations, including the estimate of $C^{*} \approx 0.95 \mathrm{wt} \%$, are given in Section S2.1 of Supporting Information.

To obtain SCNPs, first, the conditions for the successful electrospraying had to be optimized. At too high polymer concentration $(2.3 \mathrm{wt} \%)$, when $C>C^{*}$, a continuous whipping jet of polymer solution was observed and resulted in the usual electrospinning of nanofibers (NFs) as observed by SEM. The obtained NFs had a diameter of more than $100 \mathrm{~nm}$. When the concentration of the polymer solution was reduced to $C \approx C^{*}$, a mixture of particles and fibers was obtained. At $0.1 \mathrm{wt} \%$, the initial linear jet of polymer solution broke up in $27 \mu \mathrm{m}$ droplets of polymer solution within the first ca. $3 \mathrm{~mm}$ from the nozzle as observed in Figure 1. These large droplets are known to undergo secondary splitting in flight, ${ }^{33}$ resulting 
in the collected NPs containing single chains, as observed by SEM.

Differences between the obtained structures can be attributed to a combination of the state of the polymers in solution and physical properties of the solutions. Above $C^{*}$, because of entanglements present due to the mutual polymer chain overlap, and of the resulting solution viscosity, continuous jets are formed leading to electrospinning of long polymer fibers. At concentrations below $C^{*}$, the viscosity of the polymer solution is decreased, and consequently, the same shear force created by the difference of potential can lead to a larger deformation at the nozzle. This steady jet of polymer solution subsequently breaks to form droplets which will undergo secondary splitting later in-flight because of Coulombic and surface tension driven fission, yielding the NPs. However, at intermediate concentrations, close to $C^{*}$, the system was in a transition state and a mixture of structures is obtained, as observed by electron microscopy. ${ }^{27,33-35}$

Beyond direct visualization by electron microscopy, the size of single chain NPs was verified using atomic force microscopy (AFM). Figure 2a shows an AFM image of NPs prepared from

(a)
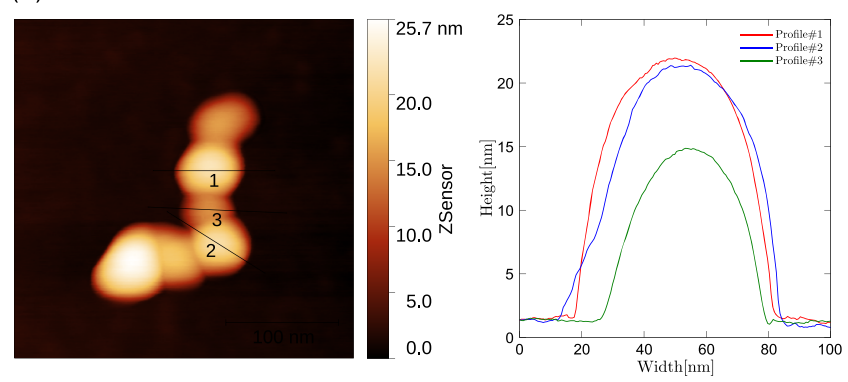

(b)

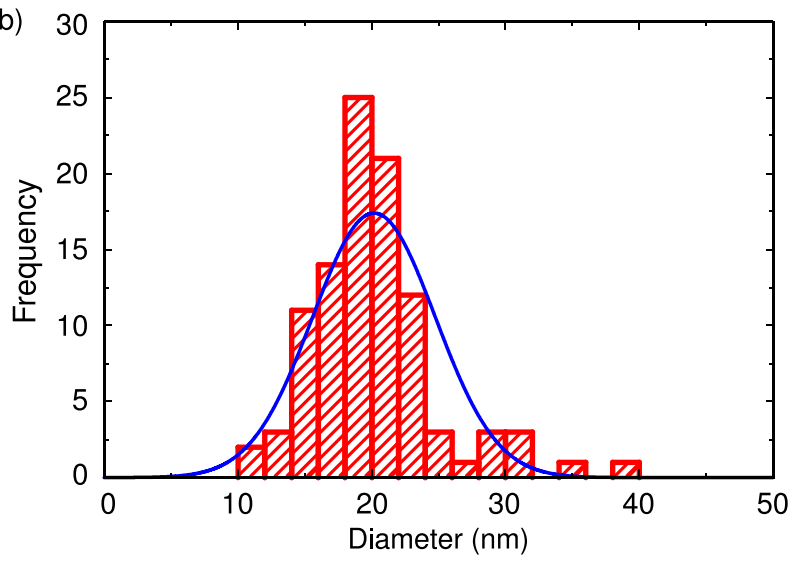

Figure 2. Size of PS SCNPs: (a) characteristic AFM image (right) of PS NPs with indicated cross-section lines to obtain the height profiles (left), and (b) size distribution of PS NPs as estimated from SEM images. Samples were prepared using electrospraying of PS in DMF solution at concentrations $C=0.1 \mathrm{wt} \%$, distance $d=20 \mathrm{~cm}$, infuserate $v=1.5 \mathrm{~mL} / \mathrm{h}$, and applied voltage of $V=15 \mathrm{kV}$.

low concentration ( $C=0.1 \mathrm{wt} \%$ ) PS in DMF solution. From the profile of different NPs, height and apparent width of the particles were found to be in the range of 15-20 and 55-60 $\mathrm{nm}$, respectively. Taking into account, that the measurement of the apparent width of the particles includes the convolution of the diameter of particles + diameter of cantilever-tip, AFM height profiles provide the most accurate measure of the size of the NPs. We further analyzed the SEM image of NPs by measuring the size distribution of $(n=100)$ randomly selected particles in Figure 1. The histogram (Figure 2b) shows that the average size of the particles is $20 \pm 3 \mathrm{~nm}$. Assuming melt density of PS, ${ }^{36}$ the diameter of a single chain NP of PS of $M_{n}$ $=1.1 \times 10^{6} \mathrm{Da}$ should be approx. $20 \mathrm{~nm}$. Details of the calculation are given in Section S4 of the Supporting Information. Thus, we can safely conclude that our NPs typically contain only one polymer chain. We also varied the infuse-rate and applied voltage, but no significant changes were observed in the NP size (Sections S4.1 and S4.2 of the Supporting Information).

\section{GLASS TRANSITION: DSC AND OSCILLATORY RHEOLOGY}

First, we compare the apparent glass transition temperature $T_{\mathrm{g}}$ of different entangled and disentangled melts of PS prepared from the very same batch of polymer and by the same cooling rate. Figure 3 shows the comparison of the glass transition temperature obtained using two different techniques. DSC results of four PS samples are shown: equilibrated bulk PS film, PS NFs, PS SCNPs, and annealed SCNPs. The bulk PS film was prepared by hot pressing $300 \mathrm{mg}$ of PS flakes under a load of $20 \mathrm{kN}$ at $433 \mathrm{~K}$ for $1 \mathrm{~h}$. The PS film had a thickness of 100 $\pm 10 \mu \mathrm{m}$. The results show that the observed glass transition temperature $\left(T_{\mathrm{g}}\right)$ of entangled samples (PS films and NFs) are the same within the error (approx. $381 \mathrm{~K}$ ), despite the strong deformation of the long chains in the fibers. This is remarkable by itself but beyond the scope of the present work. In comparison to that, the apparent $T_{\mathrm{g}}$ of the melt of disentangled PS NPs (approx. $374 \mathrm{~K}$ ) is reduced by about $2 \%$, that is, $7^{\circ}$. After annealing the SCNP sample at $433 \mathrm{~K}$ for $24 \mathrm{~h}, T_{\mathrm{g}}$ increased to $380 \mathrm{~K}$, similar to $T_{\mathrm{g}}$ of bulk entangled PS. This increase of $T_{\mathrm{g}}$ following annealing might be attributed to the development of entanglements in the disentangled SCNP sample, even though polymer globules are known to be metastable. ${ }^{17,37,38}$ Thus, the question arises, can the apparent lower $T_{\mathrm{g}}$ be attributed to the higher chain mobility in the disentangled melt in the absence of large number of entanglements? The density of chain ends is the same in each sample. It should be noted that there are no detectable traces of solvent left in the material, see Section S2.2 of the Supporting Information. We have also repeated 10 cycles of heating and cooling of PS NPs (Figure S12 in Supporting Information). The graphs showing 10 heating curves are presented in Section S5 of the Supporting Information. The result shows that the apparently lower $T_{\mathrm{g}}$ of PS NPs was quite stable and did not change significantly even after 10 cycles of heating and cooling in the range $133-473 \mathrm{~K}$ at a rate of $10 \mathrm{~K} /$ $\mathrm{min}$, that is a total of $680 \mathrm{~min}$ of experiment duration. Though this is significantly longer than the expected overall Rouse time, which is expected to govern the internal relaxation dynamics of the nonentangled particles, no signature of reentanglement is observed. Experimental ${ }^{17,18,39,40}$ and theoretical works ${ }^{37,38}$ on disentangled melts of polymers showed that chains longer than a critical length need to overcome a significant free energy barrier in order to relax from a disentangled state to a fully entangled state. The PS used in our experiments would have $z=M_{\mathrm{n}} / M_{\mathrm{e}} \approx 66$ entanglements per chain (considering $M_{\mathrm{n}}=1.1 \times 10^{6} \mathrm{Da}$ and $M_{\mathrm{e}}=16,600$ $\mathrm{Da}^{41}$ ) in a fully equilibrated entangled melt. Thus, heating of our SCNPs of long PS chains is expected to lead to the formation of metastable melts. 
(a)

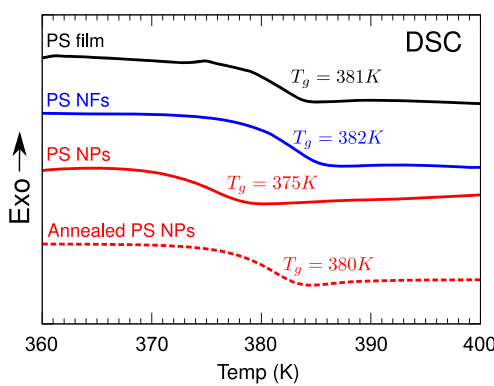

(b)

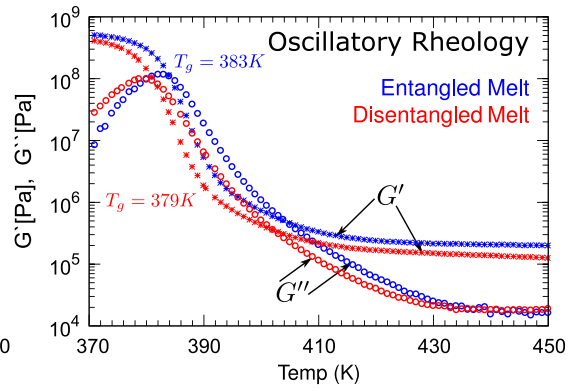

Figure 3. Comparison of the glass transition temperature $T_{\mathrm{g}}$ of entangled and disentangled PS: (a) DSC experiments: PS NPs, PS NFs, and PS film. PS NPs and NFs were prepared by electrospinning of PS in DMF solution at concentrations $C=0.2 \mathrm{wt} \%$ and $C=2.3$ wt $\%$ using the process parameters: distance $d=20 \mathrm{~cm}$ and infuse-rate $v=1.5 \mathrm{~mL} / \mathrm{h}$ and applied voltage $V=15 \mathrm{kV}$. PS film was prepared by hot-pressing bulk PS at temperature $T=433 \mathrm{~K}$ under a load of $L=20 \mathrm{kN}$. (b) Rheological experiment: temperature sweep at frequency $f=10 \mathrm{rad} / \mathrm{s}$ was used.

To examine this, Figure $3 \mathrm{~b}$ shows the temperature sweep rheology experiments on entangled and disentangled (SCNPs) PS. The loss modulus $\left(G^{\prime \prime}\right)$ measures energy dissipation, and the temperature of the peak value of $G^{\prime \prime}$ during temperature sweep gives an estimate of the glass transition temperature of polymers. ${ }^{42}$ Our oscillatory rheology experiments show an apparent lower glass transition temperature of disentangled PS NPs $\left(T_{\mathrm{g}}=379 \mathrm{~K}\right)$ in comparison to entangled PS $\left(T_{\mathrm{g}}=383\right.$ $\mathrm{K})$ in good agreement with DSC. The difference in $T_{\mathrm{g}}$ of entangled and disentangled samples from rheological experiments turns out to be smaller than that observed in DSC experiments. Note that by measuring $T_{g}$ with oscillatory rheology, we probe local relaxation rather than the overall viscosity of the melts. As in the DSC experiments, lower $T_{\mathrm{g}}$ of disentangled PS NPs was very stable. The samples for rheology experiments were prepared at a temperature of $433 \mathrm{~K}$ and the data presented in Figure 3 are from the cooling cycle after the first cycle of heating the sample to $473 \mathrm{~K}$ from room temperature. We note here that for both entangled and disentangled PS the measured values of the storage moduli in the glassy state are lower than the expected nominal value of $G^{\prime} \sim 1 \mathrm{GPa}$. This is most likely related to the effect of the device compliance that is a common problem when measuring rheological properties of a glassy material with parallel plate geometries. ${ }^{43-45}$ Nevertheless, device compliance affects in similar way the data do for all studied samples. Thus, the oscillatory rheology experiments not only reveal a slightly lower glass transition temperature for disentangled PS but also a somewhat lower dissipation and storage modulus compared to the entangled melts. These results are pointing toward a generally weaker monomer-monomer viscoelastic coupling between the polymer strands. Whether this is mainly due to interactions between NPs or to the entanglement structure, it cannot be decided based on these experiments.

\section{GLASS TRANSITION: BLS}

BLS is a powerful technique for studying dynamics ${ }^{46}$ and glass transition of amorphous materials. ${ }^{4-50}$ For transparent films, BLS measures the longitudinal modulus $L(\omega)$ at $\mathrm{GHz}$ frequencies, $\omega$. The dispersion, $L(\omega)$, in amorphous polymers is controlled by the main glass-rubber relaxation time, $\tau$, and attains the limiting low and high frequency values $L_{0}$ for $(\omega \tau)^{2}$ $\ll 1$ and $L_{\infty}$ for $(\omega \tau)^{2} \gg 1$, respectively. ${ }^{46,49}$ Assuming a typical Williams-Landel-Ferry temperature dependence for $\tau(T)$, BLS measures $L_{\infty}$ in the glassy and rubbery states at temperatures as high as $T_{\mathrm{g}}+100 \mathrm{~K}^{46}$ The temperature dependence of $L(T)=\rho(T) c^{2}(T)$, with $\rho(T)$ and $c(T)$ being the density and longitudinal sound velocity, is mainly controlled by $c(T)$ in the vicinity of $T_{\mathrm{g} .}{ }^{47-50}$ The different temperature dependences, $c\left(T<T_{\mathrm{g}}\right.$ ) (weak) and $c\left(T>T_{\mathrm{g}}\right)$ (strong), enables the determination of $T_{\mathrm{g}}$ in amorphous bulk polymers by BLS. ${ }^{47,49}$ In the case of non-transparent powder of polymeric colloids, BLS determines the glass transition through the temperature-sensitive particle elastic vibrations which disappear upon the formation of the contiguous film at $T_{\mathrm{g}}$. In addition, BLS identifies a second so-called softening temperature, $T_{\mathrm{s}}\left(<T_{\mathrm{g}}\right)$, which is related to the onset of particle surface mobility below $T_{\mathrm{g}}{ }^{51,52}$ Because the BLS intensity strongly decreases with the NP diameter, the fundamental quadrupolar vibration mode, $(1,2)$, cannot be resolved for NP diameter below about $100 \mathrm{~nm}$. In the presence of NP adhesion, however, a new interaction-induced branch $(1,1)$ representative of few NPs vibration is discernible in the BLS spectrum at frequencies lower than the $(1,2)$ mode. ${ }^{53}$

For the present non-transparent as-prepared SCNPs powder, the $(1,2)$ mode of the individual SCNP cannot be resolved because of their small size $(20 \pm 3 \mathrm{~nm})$. Figure $4 \mathrm{a}$ shows the reduced [intensity $(I)$ times frequency $(f)$ squared] BLS spectra of as-prepared SCNPs at different temperatures increasing from 294 to $373 \mathrm{~K}$. The $(1,1)$ mode is resolved as anticipated because of the SCNP adhesion in the SEM image (Figure 1). Upon this first heating, the $(1,1)$ mode undergoes a blue shift (increase of its frequency) at the softening temperature, $T_{\mathrm{s}} \approx 345 \mathrm{~K}$, and then abruptly changes in the temperature region between 363 and $373 \mathrm{~K}$ as indicated by the dashed line in Figure 4a. The strong peak in the BLS spectrum at $373 \mathrm{~K}$ is reminiscent of the longitudinal acoustic (LA) phonon in bulk PS at backscattering (BS) and hence indicates the formation of a contiguous film of molten SCNPs. The BLS spectra of the latter are presented in Figure $4 \mathrm{~b}$. The interaction-induced $(1,1)$ mode is associated with the acoustic phonon propagation in SCNP clusters of granular type (lower inset to Figure $4 \mathrm{~d}$ ), and its frequency is sensitive to the interparticle adhesion force. ${ }^{51,53}$ The position of the $(1,1)$ mode is represented by the frequency $f_{(1,1)}$ at maximum intensity in the reduced BLS spectra in Figure $4 a$, and its temperature dependence is plotted in Figure 4d (black solid circles). As temperature increases above $T_{\mathrm{s}}$ (patterned area), $f_{(1,1)}$ crossovers to the LA phonons in the film of molten SCNPs (upper inset to Figure 4d) in the region 363-373 K. Hence, the $T_{\mathrm{g}}$ of the SCNPs should fall into this temperature range (yellow shaded area in Figure $4 \mathrm{~d})$ and is slightly $(\approx 6 \mathrm{~K})$ 

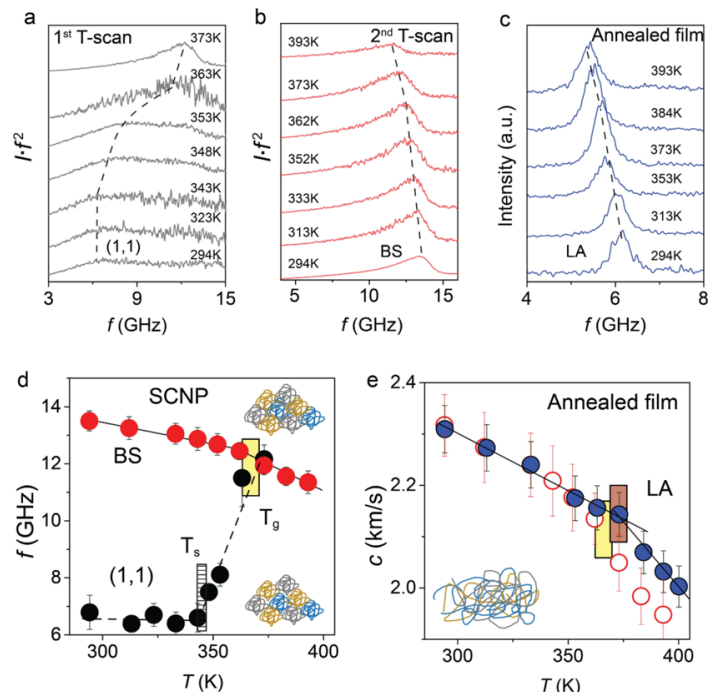

Figure 4. BLS spectra of SCNPs in three different morphologies. Evolution of the reduced BLS spectra, $I \cdot f^{2}$ vs $f$, with temperature $(T)$ for (a) the as-prepared SCNPs sample (1st T-scan) and (b) the coalescent SCNPs obtained after quenching of the as-prepared SCNP sample in (a) from $373 \mathrm{~K}$ (2nd T-scan), (c) BLS spectra ( $I$ vs $f$ ) of the transparent annealed PS film obtained after annealing under vacuum at $423 \mathrm{~K}$ for $24 \mathrm{~h}$ of the coalescent SCNP film in (b). The dashed lines indicate the variation of the frequency $f_{(1,1)}$ of $(1,1)$ mode in (a) and longitudinal phonon $f$ at scattering angle $180^{\circ}$ in (b) and at $90^{\circ}$ in (c) with $T$. The dashed lines in $(\mathrm{a}-\mathrm{c})$ are guides to the eye. (d) $T$ dependence of $f_{(1,1)}$ (black solid symbols) in the as-prepared SCNPs sample in (a) and the frequency of LA (red solid symbols) in the coalescent SCNPs sample in (b). The lower and upper insets indicate the schematics of the as-prepared SCNPs and the coalescent SCNPs. (e) $T$ dependence of the longitudinal sound velocity, $c$, of the transparent annealed film (blue solid symbols) obtained from the BLS spectra in (c) and the coalescent SCNPs of the 2nd T-scan (open red symbols) obtained from the $f$ in (d).The solid lines denote the linear $c(T)$ below and above $T_{\mathrm{g}}$. The patterned and shaded areas denote the softening temperature $T_{s}$ in the 1 st $\mathrm{T}$-scan and the glass transition temperature $T_{\mathrm{g}}$ for the three samples, respectively.

lower than the $T_{\mathrm{g}}(=374 \pm 3 \mathrm{~K})$ of the film composed of entangled PS chains (Section S6 of the Supporting Information). In this context, we should also note that the presence of $T_{s}$ indicates an increased mobility of the free SCNP surface, activating surface contacts at $T<T_{\mathrm{g}}{ }^{51}$ This surface mobility might lead to the slightly lower apparent $T_{\mathrm{g}}$ of the SCNPs (large surface/volume ratio).

To check whether the observed small shift in $T_{\mathrm{g}}$ is associated to SCNP free surface or to the missing entanglements, we compare the BLS spectra of the contiguous film composed of coalescent SCNPs (Figure 4b), after the first heating up to 373 $\mathrm{K}$ (Figure $4 \mathrm{a}$ ), and the annealed film of entangled PS chains (Figure 4c). To relax the disentangled PS chains in the SCNPs, the coalescent SCNPs sample is annealed for $24 \mathrm{~h}$ under vacuum at $T=423 \mathrm{~K}$ leading to a fully transparent film. The reduced BLS spectra from the coalescent SCNPs in a second heating cycle are shown in Figure $4 \mathrm{~b}$. The single LA mode in the BS spectrum indicates a rather homogeneous film at all examined temperatures, even though the line shape is clearly broader than for the fully annealed film in Figure 4c. The frequency of the LA phonon at the maximum of the single peak (Figure 4b) is plotted in Figure 4d (red solid circles), indicating a similar $T_{\mathrm{g}}$ (yellow shaded area) as for the asprepared SCNPs (black solid circles). The BLS spectra of the annealed film recorded at different temperatures at wave vector $q=0.0167 \mathrm{~nm}^{-1}$ are shown in Figure $4 \mathrm{c}$. The sound velocity, $c$ $=2 \pi f / q$, obtained from the frequency $f$ at the maximum of the LA mode in the annealed film, is plotted as a function of temperature in Figure 4e (blue solid circles). For comparison, Figure $4 \mathrm{e}$ includes the sound velocity (red open circles) of the LA in the coalescent SCNPs obtained from the BS frequencies (solid red symbols in Figure $4 \mathrm{~d}$ ) at $q=4 \pi n / \lambda=0.0366 \mathrm{~nm}^{-1}$, where $\lambda(=532 \mathrm{~nm})$ is the laser wavelength and $n(=1.55)$ is the refractive index. The change of the decrease rate of $c(T)$ in the annealed film occurred at $T_{\mathrm{g}}=373 \pm 2 \mathrm{~K}$ (red shaded area in Figure 4e). This is identical to $T_{\mathrm{g}}$ of the bulk PS of fully entangled chains (Supporting Information, S6) and slightly higher than that of the coalescent SCNP film (yellow shaded in Figure $4 \mathrm{e}$ ). Note that because of different mechanisms and heating rates, the $T_{\mathrm{g}}$ estimated by BLS can be about 7-10 K lower than the corresponding DSC value (Figure $3 \mathrm{a}) .^{49-51}$

The BLS results corroborate the notion that the $T_{\mathrm{g}}$ of the asprepared and coalescent SCNPs samples with disentangled chains is slightly $(\approx 6 \mathrm{~K})$ lower than that of the annealed film with entangled chains. The conceivable $T_{\mathrm{g}}$ suppression effect due to the free surface softening ${ }^{54}$ is not supported by the similar $T_{\mathrm{g}}$ values in the as-prepared and coalescent SCNPs samples (Figure 4d). Based on this similarity, the local chain structure in these two samples should assume a similar local structure. On a larger length scale, the chain packing in the experimental samples might be not the same compared to the equilibrated structures of the MD simulations. ${ }^{55,56}$

\section{SIMULATIONS}

Despite three different experiments, addressing qualitatively different quantities to locate the glass transition of the two different polymer systems, the conclusion as to whether $T_{\mathrm{g}}$ for entangled and disentangled long chain polymer melts is the same or not is not entirely clear. To complement this, we resort to simulations studies. By looking at model systems, simulations have the clear advantage to study perfectly defined systems. This comes with the disadvantage that the cooling rate which can be reached in simulations typically is much higher than in experiments. Because we follow exactly the same cooling protocol for both systems, for the current study, this is not a problem. If there is a difference in $T_{g}$, very fast cooling is expected to increase this difference. Here, we employ MD simulations, which are a very powerful tool to understand the structural and thermal behavior of polymer melts. We study a recently developed coarse-grained model, ${ }^{57,58}$ which does not suffer from artificial chain straightening under cooling and performed $\mathrm{MD}$ simulations in the isothermal-isobaric ensemble (NPT). To compare entangled and disentangled melts, we follow a strategy similar to experiment and compare a melt of fully equilibrated chains to a melt of collapsed, disentangled globules. To produce a disentangled melt, we start with isolated good solvent chains, which are contracted on a time scale much faster than the chain relaxation time. These collapsed chains are squeezed into a box and relaxed for a short time, in order to obtain a melt of SCNPs with an overall homogeneous monomer density. Figure 5 illustrates the two different systems. For details of the model and the sample preparation, we refer to Supporting Information.

We compare two systems, where the local monomer packing is identical. The only difference is that topological constraints in the form of entanglements are present in the equilibrated melt and (almost completely) absent in the melt of polymer 
(a)

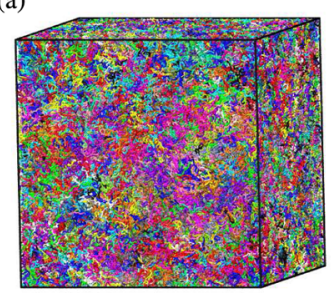

(b)

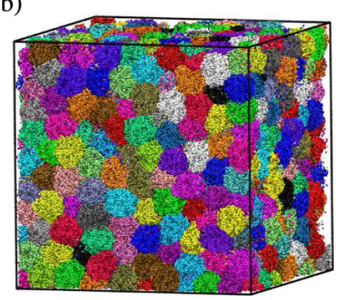

Figure 5. Snapshots of the two different polymer melts of an equilibrated system (a) and a melt of individually collapsed, disentangled chains (b) of the very same length. Each system contains 1000 chains of chain length $N=1000$ with the monomer density $\rho=0.85 \sigma^{-3}$.

globules, as also a primitive path analysis (PPA) reveals. To compare the glass transition, both systems were cooled down following exactly the same cooling protocol. Because we focus on potential differences in $T_{\mathrm{g}}$ rather than the exact value of $T_{\mathrm{g}}$, this is an adequate approach. The cooling rate of $\Gamma=8.3 \times$ $10^{-7} \epsilon /\left(k_{\mathrm{B}} \tau\right)$ was chosen such that around the highest temperature $T=1 \epsilon / k_{\mathrm{B}}$, subchains of the order of $N_{\mathrm{e}}$ easily can relax. The observed glass transition temperature $T_{\mathrm{g}}$ was obtained from the change of volume $V$ as a function of temperature. $^{59}$ The intersection of the linear extrapolation of $\ln V(T)$ between the liquid branch $\left(\ln V_{\text {liquid }}=a_{\text {liquid }}+\alpha_{\text {liquid }} T\right)$ and the glass branch $\left(\ln V_{\text {glass }}=a_{\text {glass }}+\alpha_{\text {glass }} T\right)$ can be used to estimate $T_{\mathrm{g}}$. Here, $\alpha_{\text {liquid }}$ and $\alpha_{\text {glass }}$ are thermal expansion coefficients for polymer melts in the liquid state and the glassy state, respectively. Furthermore, we checked whether the melt of globular chains did not significantly re-entangle during the simulation by performing a PPA. The longest straight pieces in PPA have lengths of the order of the whole chains, proving that the disentangled globules remain intact during the simulation. Results of $\ln V$ plotted versus $T$ for both entangled (with the entanglement length $N_{\mathrm{e}}=28$ obtained from the $\mathrm{PPA}^{60}$ of chains with bending parameter, $k_{\theta}=1.5^{61,62}$ used in the current work) and disentangled polymer melts each containing 1000 chains of polymerization degree $N=1000$ with a starting monomer density $\rho=0.85 \sigma^{-3}$ are shown in Figure $6 \mathrm{a}$. The glass transition occurs at $T_{\mathrm{g}}=0.670(2) \epsilon / k_{\mathrm{B}}$ for the disentangled and the entangled polymer melt at pressure $P=$ $0.0 \epsilon / \sigma^{3}$. For comparison, for a melt of short chains of $N=50$ and the identical quench rate, we find $T_{\mathrm{g}}=0.638(4) \epsilon / k_{\mathrm{B}}{ }^{57,58}$
It is obvious from Figure 6 that there is no detectable difference in $T_{\mathrm{g}}$ for the two systems. Also, the densities of the two different systems are almost indistinguishable $(\rho=$ $\left.0.95 \sigma^{-3}\right)$. Furthermore, the monomer packing, as shown by the radial nonbonded monomer-monomer distribution function $g(r)$ shown in Figure $6 \mathrm{~b}$, reveals that the local melt structures are identical. A $T_{\mathrm{g}}$ shift of about $2 \%$ as in the initial DSC experiment certainly would have been detected. Also, the different contributions to the energy of the melts are indistinguishable, as revealed by Figure 7 for the bonded (a) and the nonbonded part (b). To observe any detectable deviation, we have to closely look at individual contributions to the energy. Figure $7 \mathrm{c}$ shows the temperature dependence of the repulsive part of the bonded interaction along the backbone. Up to a very small shift in absolute value, the shapes are identical all the way up to tiny details. Thus, there are no observable compensation effects from different contributions, which might be affected differently by the presence/lack of entanglement constraints.

\section{CONCLUSIONS}

In this study, we have compared the glass transition of melts of highly entangled polymers to that of disentangled polymers of the very same molecular weight by three different experimental techniques and computer simulations. Chains in experiments contain about $66 M_{e}$ based on an entanglement molecular weight of $M_{e}=16,600 \mathrm{Da},{ }^{41}$ while the chain length in our simulation study corresponds to about $36 N_{\mathrm{e}}$. Thus, in equilibrium, both systems are in the fully entangled regime. In both, simulations and experiments, great care has been taken to produce films/systems of disentangled SCNPs. For the simulations, this is confirmed by a PPA. From the simulations, we know that the local packing of the monomers in both cases is identical and that we study a sample with a homogeneous density. Thus, in contrast to the experiments, the simulation systems are perfectly characterized. Both a careful analysis of the specific volume during the temperature change and a very detailed analysis of different contributions to the energy do not reveal any change in $T_{\mathrm{g}}$ or of behavior around $T_{\mathrm{g}}$ of any other tested property. For the experimental analysis, this is less obvious. DSC and oscillatory rheology suggest a $T_{\mathrm{g}}$ shift, which is comparable to the difference in $T_{\mathrm{g}}$ between chains of a molecular weight of about $M_{\mathrm{e}}$ and that of
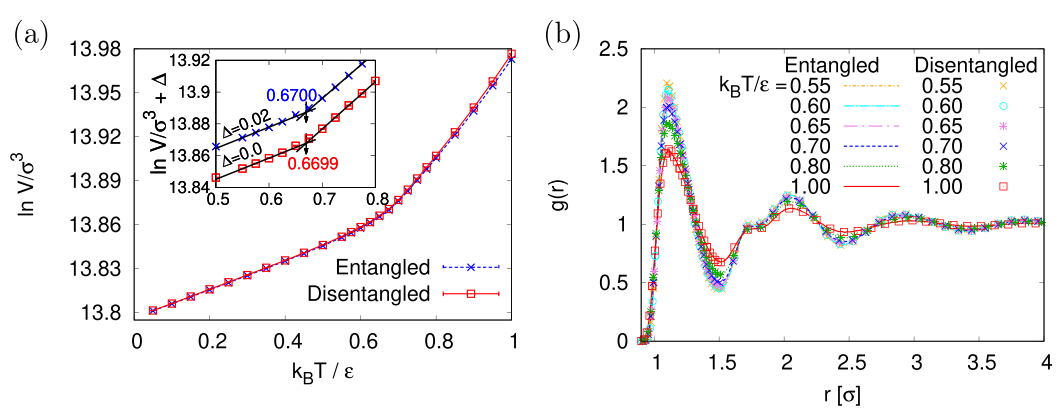

Figure 6. MD simulations: (a) logarithm of volume of the system, $\ln V / \sigma^{3}$, plotted vs temperature $k_{\mathrm{B}} T / \epsilon$. (b) Radial nonbonded monomermonomer distribution function $g(r)$ for disentangled and entangled polymer melts. Several values of $T$ are chosen, as indicated. In (a), the two linear lines give the best fit of our data along the liquid branch $\left(a_{\text {liquid }}^{\text {(disent) }} \approx 13.6681, \alpha_{\text {liquid }}^{\text {(disent) }} \approx 0.2981 k_{\mathrm{B}} / \epsilon\right),\left(a_{\text {liquid }}^{(\text {ent })} \approx 13.6733, \alpha_{\text {liquid }}^{\text {(ent) }} \approx 0.2896 k_{\mathrm{B}} / \epsilon\right)$ and the glass branch $\left(a_{\text {glass }}^{\text {(disent) }} \approx 13.7784, \alpha_{\text {glass }}^{\text {(disent })} \approx 0.1333 k_{\mathrm{B}} / \epsilon\right),\left(a_{\text {glass }}^{\text {(ent })} \approx 13.7776, \alpha_{\text {glass }}^{\text {(ent) }} \approx 0.1339 k_{\mathrm{B}} / \epsilon\right)$ for disentangled and entangled polymer melts, respectively. The error bars of the data are within the size of symbols. In the inset data for the same quench rate but a much smaller $T$ range are given for comparison. Data for the entangled polymer melt are shifted by a constant $\Delta=0.02$ for a better illustration. Confined to that small interval the shift in $T_{\mathrm{g}}$ diminishes. 
(a)

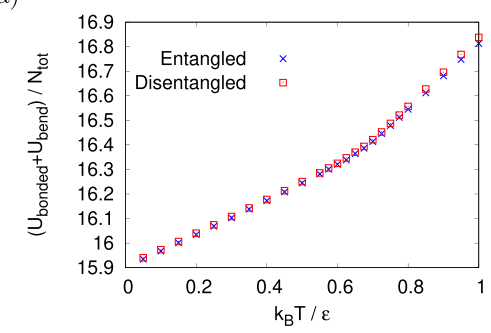

(b)

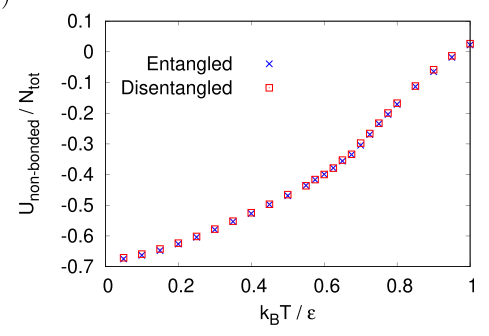

(c)

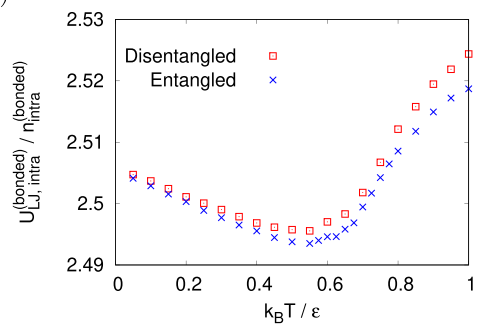

Figure 7. Temperature dependence of the average bonded and bending (a) and nonbonded (b) potential energies per monomer and repulsive part of the bonded potential energy per monomer pairs (c).

highly entangled chains. Using eq 1 to compare PS of $M_{e}=M_{n}$ $=16,600 \mathrm{Da}$ and $M_{\mathrm{n}}=1.1 \times 10^{6} \mathrm{Da}$, a difference of approximately $6 \mathrm{~K}$ in $T_{\mathrm{g}}$ is expected ${ }^{3,7}$ which is very close to the shift observed in our experimental study. In contrast to the simulation results, such an agreement points to a possible critical role of entanglements in this context. The lower $T_{\mathrm{g}}$ of the disentangled melt of high molecular weight PS SCNPs was very stable and recovered the state of entangled melts only after annealing for $24 \mathrm{~h}$ at a temperature of $T=433 \mathrm{~K}$, much higher than $T_{\mathrm{g}}$ of PS. The heterogeneous distribution of entanglements makes the relaxation of disentangled melts very slow. This delay originates from the deformation of the heterogeneous network (of entangled and disentangled regions) in the course of relaxation. ${ }^{37}$ To shed even more light onto this dichotomy, BLS was performed on the very same systems. Upon the first and second heating, a $T_{\mathrm{g}}$ shift has been observed as well. Unlike for the simulations, it is not possible to continuously control the level of entanglement during the different cycles in the experiments. The fully annealed sample certainly produces the same $T_{\mathrm{g}}$ as the fully entangled melt. In summary, these results suggest that local packing dominates the glass transition process and that topological constraints due to chain entanglements do not play a significant role. On the other hand, it is known that the disentangled state is long lived and that such a melt does not display a viscoelastic plateau modulus. Thus, to shed more light on potential viscoelastic inhomogeneities, further experiments and computer simulations are needed. It will also be interesting to perform stress-relaxation experiments on the SCNPs and compare the same with that of entangled melt of linear chains and also with the melt of ring polymers. This should shed even more light on the stability of SCNPs and their physical properties.

\section{METHODOLOGY}

Electrospraying. PS was dissolved in varying amounts of DMF and mechanically stirred overnight to prepare the PS in DMF solution with different concentrations of $0.1,0.5$, and 2.3 wt $\%$. The resulting solutions were filled into a syringe and then electrosprayed at $20-25 \%$ relative humidity and room temperature onto an aluminum foil support. The diameter of the tip of the needle is $0.8 \mathrm{~mm}$. The distance between the collector and tip is $20 \mathrm{~cm}$. The feeding rate was varied from 0.5 to $1.5 \mathrm{~mL} / \mathrm{h}$. The applied voltage was varied from 11 to $19 \mathrm{kV}$. To prepare SCNPs in larger quantity, a 0.2 wt \% PS in DMF solution was electrosprayed.

Scanning Electron Microscopy. The electrosprayed samples were collected on aluminum foils during electrospraying process for SEM imaging. The morphology of electrosprayed samples was observed by SEM (Hitachi SU8000, Japan).

Atomic Force Microscopy. For AFM, the measurement device (Dimension Icon with ScanAsyst) was operated under tapping mode in air. The cantilever (NanoWorld Tapping Mode AFM Probe, Model: ARRO-NCR) used had a resonant frequency of $285 \mathrm{kHz}$ and a spring constant of $42 \mathrm{~N} / \mathrm{m}$. Such cantilevers have reflective aluminum coating on the back side. For AFM, samples were collected on a silicon wafer for $30 \mathrm{~s}$ only to avoid multilayer formation of NPs. We further focused on a small area having a monolayer of NPs.

Differential Scanning Calorimetry. All DSC experiments were carried out on a 204F1/ASC Phönix (Netzsch, nitrogen flow of $20 \mathrm{~mL} / \mathrm{min}$ ) at heating and cooling rates of $10 \mathrm{~K} / \mathrm{min}$. Only heating cycles are reported.

Oscillatory Rheology. Oscillatory rheology experiments were performed using an ARES (TA Instruments). Oscillatory shear deformation was applied with controlled deformation amplitude in the range from 0.02 to $1 \%$ such that it was kept in the range of the linear viscoelastic response. Plate-plate geometry was used with a plate diameter of $6 \mathrm{~mm}$. The rheology experiments were carried out on cylindrical pellet samples of PS (one made of bulk PS flakes and other one of SCNPs) with a radius of $3 \mathrm{~mm}$ and a thickness of $1 \mathrm{~mm}$. The pellets were prepared by hot-pressing $30 \mathrm{mg}$ of PS at $433 \mathrm{~K}$ under $5 \mathrm{kN}$ load. Experiments were performed in a dry nitrogen atmosphere in the temperature range between 363 and $473 \mathrm{~K}$. Oscillatory rheology spectra (values of storage $\left(G^{\prime}\right)$ and loss $\left(G^{\prime \prime}\right)$ shear modulus as well as loss tangent $(\tan \delta=$ $\left.G^{\prime \prime} / G^{\prime}\right)$ as a function of temperature were obtained at a radial frequency $f=10 \mathrm{rad} / \mathrm{s}$. The data were obtained at various 
constant temperatures maintained to within $\pm 0.1 \mathrm{~K}$ and at a heating/cooling rate of $2 \mathrm{~K} / \mathrm{min}$.

Brillouin Light Spectroscopy. BLS is a nondestructive technique for probing the thermal density fluctuations of a material by measuring the inelastic scattered light from thermally-activated phonons. The probing wave vector $q=k_{\mathrm{s}}$ $-k_{\mathrm{i}}$ is defined as the difference between scattering light $k_{\mathrm{s}}$ and incident light $k_{\mathrm{i}}$. In the transparent media, such as a PS film, the probing wavevector is selected with the scattering geometry. We used transmission geometry to record the spectra of transparent annealed PS films, where $q=\frac{4 \pi}{\lambda} \sin \frac{\theta}{2}$ is independent of the refractive index; $\lambda=532 \mathrm{~nm}$ is the wavelength of incident light, and $\theta$ is the scattering angle. The BLS spectra consist of a doublet with a Doppler frequency shift with magnitude $f= \pm c q / 2 \pi$ at a given $q$, where $c$ is the sound velocity in the media. In the turbid media, such as fresh SCNPs, $q$ is ill-defined because of the strong multiple light scattering. Consequently, the BLS spectrum reveals the resonance modes of the clusters, uniquely defined by the geometric, elastic characteristics, and adhesion of particles. In temperature-controlled BLS experiments, the temperature is monitored with platinum resistance temperature detectors and controlled within $\pm 0.5 \mathrm{~K}$ of the set-point value with a custom temperature controller. Samples are isothermally equilibrated at each temperature for at least $20 \mathrm{~min}$ before recording a spectrum.

Simulation. The polymer chains are described as beads (monomers) connected with springs ${ }^{57,58,63}$ (the simulation details and model parameters are given in the Section S7). This model reproduces the characteristic non-Arrhenius behavior that the viscosity of polymer melts in the liquid state dramatically increases close to $T_{g}$, while the chain conformations only marginally change upon cooling as has been observed for polymers in many experiments. The simulations were performed for both entangled ${ }^{60,64,65}$ and disentangled ${ }^{38}$ polymer melt systems with the very same interaction potentials at the same monomer density $\rho=0.85 \sigma^{-3}$. Hoover barostat with Langevin thermostat ${ }^{66,67}$ (implemented in ESPResSo+ $+{ }^{68}$ ) was used at constant temperature $T$ by a stepwise cooling $^{59}$ and constant pressure $P=0.0 \epsilon / \sigma^{3}$. Each system consisted of 1000 chains of degree of polymerization (chain length) $N=1000 \approx 36 N_{\mathrm{e}}$. Thus, chains in the entangled polymer melt system are highly entangled. The temperature was decreased in steps of $\Delta T=0.025 \epsilon / k_{\mathrm{B}}$. A relaxation time of $\Delta t=30,000 \tau=0.01 \tau_{\mathrm{R}, \mathrm{N}}\left(\tau_{\mathrm{R}, \mathrm{N}}=2.89 \times N^{2} \tau\right.$ being the Rousetime at the starting temperature $\left.T=1.0 \epsilon / k_{\mathrm{B}}\right)^{60}$ was allowed between steps. This resulted in a cooling rate of $\Gamma=\Delta T / \Delta t=$ $8.3 \times 10^{-7} \epsilon /\left(k_{\mathrm{B}} \tau\right)$, which is by far too fast for entanglement effects between chains to become relevant.

\section{ASSOCIATED CONTENT}

\section{s) Supporting Information}

The Supporting Information is available free of charge at https://pubs.acs.org/doi/10.1021/acs.macromol.0c00550.

Molecular weight distribution (GPC experiments) of the experimentally studied PS; viscosity, surface tension, and hydrodynamic radius of PS in DMF at different concentrations; TEM images of PS NPs; NMR graphs of PS NPs and NFs; TGA scan of PS NPs and bulk PS; graph showing effects of voltage and infuse-rate on size of PS NPs; simulation model; chain conformations in both entangled and disentangled polymer melts; and the collective structure of melts (PDF)

\section{AUTHOR INFORMATION}

\section{Corresponding Author}

Kurt Kremer - Max Planck Institute for Polymer Research, D55128 Mainz, Germany; 이이.org/0000-0003-1842-

9369; Email: kremer@mpip-mainz.mpg.de

\section{Authors}

Manjesh K. Singh - Max Planck Institute for Polymer Research, D-55128 Mainz, Germany; 이이.org/0000-0002-91563495

Minghan Hu - Max Planck Institute for Polymer Research, D55128 Mainz, Germany; (i) orcid.org/0000-0002-9001-6780

Yu Cang - Max Planck Institute for Polymer Research, D-55128 Mainz, Germany

Hsiao-Ping Hsu - Max Planck Institute for Polymer Research, D-55128 Mainz, Germany; 이이이.org/0000-0002-82715346

Heloise Therien-Aubin - Max Planck Institute for Polymer Research, D-55128 Mainz, Germany; 이이.org/00000003-4567-516X

Kaloian Koynov - Max Planck Institute for Polymer Research, D-55128 Mainz, Germany; 이이이.org/0000-0002-40628834

George Fytas - Max Planck Institute for Polymer Research, D55128 Mainz, Germany

Katharina Landfester - Max Planck Institute for Polymer Research, D-55128 Mainz, Germany; 이이.org/00000001-9591-4638

Complete contact information is available at:

https://pubs.acs.org/10.1021/acs.macromol.0c00550

\section{Notes}

The authors declare no competing financial interest.

\section{ACKNOWLEDGMENTS}

The authors thank Gunnar Glasser, Helma Burg and Petra Räder for their help in carrying SEM, AFM and DSC characterizations respectively. The authors also thank Christine Rosenauer for helping with DLS, GPC and viscosity experiments. The authors further thank Andreas Hanewald for his help in performing DSC and rheometry experiments. The authors thank Detlef Lohse and Maziyar Jalaal from the University of Twente for the use of their high speed imaging equipment. We also gratefully acknowledge the computing time granted by the John von Neumann Institute for Computing (NIC) and provided on the supercomputer JUROPA at Jülich Supercomputing Centre (JSC), and the Max Planck Computing and Data Facility (MPCDF). The authors also thank Arghya Dutta and Robin Cortes-Huerto from MPIP Mainz for critical reading of the manuscript. This work has been supported by the European Research Council under the European Union's Seventh Framework Programme (no. FP7/2007-2013)/ERC grant agreement no. 340906MOLPROCOMP and ERC Smartphon (no. 694977).

\section{ABBREVIATIONS}

IR, infrared; NMR, nuclear magnetic resonance; UV, ultraviolet 


\section{REFERENCES}

(1) Fox, T. G., Jr.; Flory, P. J. Second-order transition temperatures and related properties of polystyrene. I. Influence of molecular weight. J. Appl. Phys. 1950, 21, 581-591.

(2) Fox, T. G.; Flory, P. J. The glass temperature and related properties of polystyrene. Influence of molecular weight. J. Polym. Sci. 1954, 14, 315-319.

(3) Ueberreiter, K.; Kanig, G. Self-plasticization of polymers. J. Colloid Sci. 1952, 7, 569-583.

(4) Rudin, A.; Burgin, D. Effects of molecular weight and chain ends on glass transition of polystyrene. Polymer 1975, 16, 291-297.

(5) Cowie, J. M. G.; Toporowski, P. M. The dependence of glass temperature on molecular weight for poly $\alpha$-methyl styrene. Eur. Polym. J. 1968, 4, 621-625.

(6) Hintermeyer, J.; Herrmann, A.; Kahlau, R.; Goiceanu, C.; Rössler, E. A. Molecular weight dependence of glassy dynamics in linear polymers revisited. Macromolecules 2008, 41, 9335-9344.

(7) Santangelo, P. G.; Roland, C. M. Molecular weight dependence of fragility in polystyrene. Macromolecules 1998, 31, 4581-4585.

(8) Zhang, L.; Marsiglio, J. A.; Lan, T.; Torkelson, J. M. Dramatic tunability of the glass transition temperature and fragility of low molecular weight polystyrene by initiator fragments located at chain ends. Macromolecules 2016, 49, 2387-2398.

(9) Rong, W.; Fan, Z.; Yu, Y.; Bu, H.; Wang, M. Influence of entanglements on glass transition of atactic polystyrene. J. Polym. Sci., Part B: Polym. Phys. 2005, 43, 2243-2251.

(10) Huang, D.; Yang, Y.; Zhuang, G.; Li, B. Influence of entanglements on the glass transition and structural relaxation behaviors of macromolecules. 1. Polycarbonate. Macromolecules 1999, 32, 6675-6678.

(11) Rizos, A. K.; Ngai, K. L. Local segmental dynamics of low molecular weight polystyrene: New results and interpretation. Macromolecules 1998, 31, 6217-6225.

(12) Roland, C. M.; Nagi, K. L. Segmental relaxation in poly(dimethylsiloxane). Macromolecules 1996, 29, 5747-5750.

(13) Miwa, Y.; Yamamoto, K.; Sakaguchi, M.; Sakai, M.; Makita, S.; Shimada, S. Direct detection of high mobility around chain ends of poly(methyl methacrylate) by the spin-labeling. Macromolecules 2005, $38,832-838$

(14) Ellison, C. J.; Mundra, M. K.; Torkelson, J. M. Impacts of polystyrene molecular weight and modification to the repeat unit structure on the glass transition- nanoconfinement effect and the cooperativity length scale. Macromolecules 2005, 38, 1767-1778.

(15) Miwa, Y.; Urakawa, O.; Nobukawa, S.; Kutsumizu, S. Selective determination of glass transition temperature and vibrational properties at the chain end of polystyrene by Fourier transform infrared measurement in combination with deuterium-labeling. Polymer 2015, 59, 194-199.

(16) Teng, C.; Gao, Y.; Wang, X.; Jiang, W.; Zhang, C.; Wang, R.; Zhou, D.; Xue, G. Reentanglement kinetics of freeze-dried polymers above the glass transition temperature. Macromolecules 2012, 45, 6648-6651.

(17) Rastogi, S.; Lippits, D. R.; Peters, G. W. M.; Graf, R.; Yao, Y.; Spiess, H. W. Heterogeneity in polymer melts from melting of polymer crystals. Nat. Mater. 2005, 4, 635.

(18) Rastogi, S.; Kurelec, L.; Cuijpers, J.; Lippits, D.; Wimmer, M.; Lemstra, P. J. Disentangled state in polymer melts; a route to ultimate physical and mechanical properties. Macromol. Mater. Eng. 2003, 288, 964-970.

(19) Boyer, R. F.; Heidenreich, R. D. Molecular Weight Studies on High Polymers with the Electron Microscope. J. Appl. Phys. 1945, 16, 621-639.

(20) Bittiger, V. H.; Husemann, E. Elektronenmikroskopische Abbildung von Einmolekülkristallen bei Cellulosetricarbanilaten. Makromol. Chem. 1964, 75, 222-224.

(21) Bittiger, V. H.; Husemann, E. Elektronenmikroskopische untersuchungen über die bildung von einmolekülkristallen aus cellulosetricarbanilat. Makromol. Chem. 1964, 80, 239-241.
(22) Bittiger, V. H.; Husemann, E. Elektronenmikroskopische untersuchungen über die kristallisation von makromolekülen aus verdünnter lösung. 3. Über den Einfluß von molekulargewicht und konzentration auf keimbildung und kristallwachstum bei cellulosetricarbanilaten. Makromol. Chem. 1966, 96, 92-99.

(23) Koszterszitz, G.; Barnikol, W. K. R.; Schulz, G. V. Die Bestimmung der Molekulargewichtsverteilung von nichtkristallisierenden Polymeren mit dem Elektronenmikroskop, 4. Weiterentwicklung der Präparationsmethode durch Gefriertrocknung. Makromol. Chem. 1977, 178, 1133-1148.

(24) Kebarle, P.; Tang, L. From ions in solution to ions in the gas phase-the mechanism of electrospray mass spectrometry. Anal. Chem. 1993, 65, 972A-986A

(25) Barbero, D. R.; Steiner, U. Nonequilibrium polymer rheology in spin-cast films. Phys. Rev. Lett. 2009, 102, 248303.

(26) Greiner, A.; Wendorff, J. H. Electrospinning: a fascinating method for the preparation of ultrathin fibers. Angew. Chem., Int. Ed. 2007, 46, 5670-5703.

(27) Festag, R.; Alexandratos, S. D.; Cook, K. D.; Joy, D. C.; Annis, B.; Wunderlich, B. Single-and few-chain polystyrene particles by electrospray. Macromolecules 1997, 30, 6238-6242.

(28) Gupta, P.; Elkins, C.; Long, T. E.; Wilkes, G. L. Electrospinning of linear homopolymers of poly(methyl methacrylate): exploring relationships between fiber formation, viscosity, molecular weight and concentration in a good solvent. Polymer 2005, 46, 4799-4810.

(29) Zheng, W.; Simon, S. L. Polystyrene freeze-dried from dilute solution: Tg depression and residual solvent effects. Polymer 2006, 47, $3520-3527$

(30) León, S.; van der Vegt, N.; Delle Site, L.; Kremer, K. Bisphenol A Polycarbonate: Entanglement Analysis from Coarse-Grained MD Simulations. Macromolecules 2005, 38, 8078-8092.

(31) Rubinstein, M.; Colby, R. Polymer Physics; Oxford University Press: New York City, 2008.

(32) Graessley, W. W. The Entanglement Concept in Polymer Rheology; Advances in Polymer Science; Springer-Verlag, 1974; Vol. 16, pp 1-179.

(33) Jaworek, A.; Sobczyk, A. T.; Krupa, A. Electrospray application to powder production and surface coating. J. Aerosol Sci. 2018, 125, $57-92$.

(34) Eda, G.; Liu, J.; Shivkumar, S. Solvent effects on jet evolution during electrospinning of semi-dilute polystyrene solutions. Eur. Polym. J. 2007, 43, 1154-1167.

(35) Smith, R. D.; Loo, J. A.; Edmonds, C. G.; Barinaga, C. J.; Udseth, H. R. New developments in biochemical mass spectrometry: electrospray ionization. Anal. Chem. 1990, 62, 882-899.

(36) Priddy, D. Styrene Polymers. In Encyclopedia of Polymer Science and Technology; Mark, H. F., Ed.; Wiley: Hoboken, 2014; Vol. 15, pp 247-336.

(37) McLeish, T. C. B. A theory for heterogeneous states of polymer melts produced by single chain crystal melting. Soft Matter 2007, 3, $83-87$.

(38) Vettorel, T.; Kremer, K. Development of entanglements in a fully disentangled polymer melt. Macromol. Theory Simul. 2010, 19, $44-56$.

(39) Lippits, D. R.; Rastogi, S.; Höhne, G. W. H.; Mezari, B.; Magusin, P. C. M. M. Heterogeneous distribution of entanglements in the polymer melt and its influence on crystallization. Macromolecules 2007, 40, 1004-1010.

(40) Lippits, D. R.; Rastogi, S.; Talebi, S.; Bailly, C. Formation of entanglements in initially disentangled polymer melts. Macromolecules 2006, 39, 8882-8885.

(41) Fetters, L. J.; Lohse, D. J.; Colby, R. H. Chain Dimensions and Entanglement Spacings, 2nd ed.; Springer: New York, 2007; pp 447454 .

(42) Rieger, J. The glass transition temperature Tg of polymersComparison of the values from differential thermal analysis (DTA, DSC) and dynamic mechanical measurements (torsion pendulum). Polym. Test. 2001, 20, 199-204. 
(43) Laukkanen, O.-V. Small-diameter parallel plate rheometry: a simple technique for measuring rheological properties of glassforming liquids in shear. Rheol. Acta 2017, 56, 661-671.

(44) Gottlieb, M.; Macosko, C. W. The effect of instrument compliance on dynamic rheological measurements. Rheol. Acta 1982, 21, 90-94.

(45) Marin, G. In Rheological Measurement; Collyer, A. A., Clegg, D. W., Eds.; Springer Netherlands: Dordrecht, 1998; Chapter Oscillatory Rheometry, pp 3-46.

(46) Voudouris, P.; Gomopoulos, N.; Le Grand, A.; Hadjichristidis, N.; Floudas, G.; Ediger, M. D.; Fytas, G. Does Brillouin light scattering probe the primary glass transition process at temperatures well above glass transition? J. Chem. Phys. 2010, 132, 074906.

(47) Eberle, R.; Anders, S. H.; Weishaupt, K.; Pietralla, M. Anisotropic effects of the glass transition in oriented polyethylene. Europhys. Lett. 1998, 43, 201.

(48) Rivnay, J.; Toney, M. F.; Zheng, Y.; Kauvar, I. V.; Chen, Z.; Wagner, V.; Facchetti, A.; Salleo, A. Unconventional face-on texture and exceptional in-plane order of a high mobility n-type polymer. Adv. Mater. 2010, 22, 4359-4363.

(49) Cang, Y.; Reuss, A. N.; Lee, J.; Yan, J.; Zhang, J.; AlonsoRedondo, E.; Sainidou, R.; Rembert, P.; Matyjaszewski, K.; Bockstaller, M. R.; Fytas, G. Thermomechanical Properties and Glass Dynamics of Polymer-Tethered Colloidal Particles and Films. Macromolecules 2017, 50, 8658-8669.

(50) Guo, Y.; Morozov, A.; Schneider, D.; Chung, J. W.; Zhang, C.; Waldmann, M.; Yao, N.; Fytas, G.; Arnold, C. B.; Priestley, R. D. Ultrastable nanostructured polymer glasses. Nat. Mater. 2012, 11, 337.

(51) Kim, H.; Cang, Y.; Kang, E.; Graczykowski, B.; Secchi, M.; Montagna, M.; Priestley, R. D.; Furst, E. M.; Fytas, G. Direct observation of polymer surface mobility via nanoparticle vibrations. Nat. Commun. 2018, 9, 2918.

(52) Kang, E.; Graczykowski, B.; Jonas, U.; Christie, D.; Gray, L. A. G.; Cangialosi, D.; Priestley, R. D.; Fytas, G. Shell Architecture Strongly Influences the Glass Transition, Surface Mobility, and Elasticity of Polymer Core-Shell Nanoparticles. Macromolecules 2019, 52, 5399-5406.

(53) Mattarelli, M.; Montagna, M.; Still, T.; Schneider, D.; Fytas, G. Vibration spectroscopy of weakly interacting mesoscopic colloids. Soft Matter 2012, 8, 4235-4243.

(54) Zhang, C.; Guo, Y.; Priestley, R. D. Glass transition temperature of polymer nanoparticles under soft and hard confinement. Macromolecules 2011, 44, 4001-4006.

(55) Hsu, H.-P.; Kremer, K. Primitive Path Analysis and Stress Distribution in Highly Strained Macromolecules. ACS Macro Lett. 2018, 7, 107-111.

(56) Hsu, H.-P.; Kremer, K. Clustering of Entanglement Points in Highly Strained Polymer Melts. Macromolecules 2019, 52, 67566772.

(57) Hsu, H.-P.; Kremer, K. A coarse-grained polymer model for studying the glass transition. J. Chem. Phys. 2019, 150, 091101.

(58) Hsu, H.-P.; Kremer, K. Erratum: "A coarse-grained polymer model for studying the glass transition” (J. Chem. Phys. 150, 091101 (2019)). J. Chem. Phys. 2019, 150, 159902.

(59) Buchholz, J.; Paul, W.; Varnik, F.; Binder, K. Cooling rate dependence of the glass transition temperature of polymer melts: Molecular dynamics study. J. Chem. Phys. 2002, 117, 7364-7372.

(60) Hsu, H.-P.; Kremer, K. Static and dynamic properties of large polymer melts in equilibrium. J. Chem. Phys. 2016, 144, 154907.

(61) Everaers, R.; Sukumaran, S. K.; Grest, G. S.; Svaneborg, C.; Sivasubramanian, A.; Kremer, K. Rheology and microscopic topology of entangled polymeric liquids. Science 2004, 303, 823-826.

(62) Hsu, H.-P.; Kremer, K. Detailed analysis of Rouse mode and dynamic scattering function of highly entangled polymer melts in equilibrium. Eur. Phys. J.: Spec. Top. 2017, 226, 693-703.

(63) Kremer, K.; Grest, G. S. Dynamics of entangled linear polymer melts: A molecular-dynamics simulation. J. Chem. Phys. 1990, 92, 5057-5086.
(64) Zhang, G.; Moreira, L. A.; Stuehn, T.; Daoulas, K. C.; Kremer, K. Equilibration of high molecular weight polymer melts: a hierarchical strategy. ACS Macro Lett. 2014, 3, 198-203.

(65) Moreira, L. A.; Zhang, G.; Müller, F.; Stuehn, T.; Kremer, K. Direct equilibration and characterization of polymer melts for computer simulations. Macromol. Theory Simul. 2015, 24, 419-431.

(66) Martyna, G. J.; Tobias, D. J.; Klein, M. L. Constant pressure molecular dynamics algorithms. J. Chem. Phys. 1994, 101, 4177.

(67) Quigley, D.; Probert, M. I. J. Langevin dynamics in constant pressure extended systems. J. Chem. Phys. 2004, 120, 11432-11441.

(68) Halverson, J. D.; Brandes, T.; Lenz, O.; Arnold, A.; Bevc, S.; Starchenko, V.; Kremer, K.; Stuehn, T.; Reith, D. ESPResSo++: A modern multiscale simulation package for soft matter systems. Comput. Phys. Commun. 2013, 184, 1129-1149. 